\title{
Association Mapping of Flowering Time QTLs and Insight into Their Contributions to Rapeseed Growth Habits
}

\author{
Nian Wang ${ }^{1,2}$, Biyun Chen ${ }^{1}$, Kun X Xu ${ }^{1}$, Guizhen Gao ${ }^{1}$, Feng $\mathrm{Li}^{1}$, Jiangwei Qiao ${ }^{1}$, \\ Guixin Yan ${ }^{1}$, Jun $\mathrm{Li}^{1}$, Hao $\mathrm{Li}^{1}$ and Xiaoming $\mathrm{Wu}^{1 *}$ \\ ${ }^{1}$ Key Laboratory of Biology and Genetic Improvement of Oil Crops, Ministry of Agriculture, Oil Crop Research Institute of the \\ Chinese Academy of Agricultural Sciences, Wuhan, China, ${ }^{2}$ College of Horticulture and Forestry Sciences, Huazhong \\ Agricultural University, Wuhan, China
}

OPEN ACCESS

Edited by:

Christian Jung,

Christian Albrechts University of Kiel,

Germany

Reviewed by:

Swarup Kumar Parida,

National Institute of Plant Genome

Research, India

Nazgol Emrani,

Christian Albrechts University of Kiel,

Germany

Ping Lou,

Dartmouth College, USA

*Correspondence:

Xiaoming Wu

wuxm@oilcrops.cn

Specialty section: This article was submitted to Plant Genetics and Genomics, a section of the journal Frontiers in Plant Science

Received: 06 December 2015 Accepted: 04 March 2016 Published: 24 March 2016

Citation:

Wang N, Chen B, Xu K, Gao G, Li F, Qiao J, Yan G, Li J, Li H and Wu X (2016) Association Mapping of Flowering Time QTLs and Insight into Their Contributions to Rapeseed Growth Habits.

Front. Plant Sci. 7:338 doi: 10.3389/fpls.2016.00338
Plants have developed sophisticated systems to adapt to local conditions during evolution, domestication and natural or artificial selection. The selective pressures of these different growing conditions have caused significant genomic divergence within species. The flowering time trait is the most crucial factor because it helps plants to maintain sustainable development. Controlling flowering at appropriate times can also prevent plants from suffering from adverse growth conditions, such as drought, winter hardness, and disease. Hence, discovering the genome-wide genetic mechanisms that influence flowering time variations and understanding their contributions to adaptation should be a central goal of plant genetics and genomics. A global core collection panel with 448 inbred rapeseed lines was first planted in four independent environments, and their flowering time traits were evaluated. We then performed a genome-wide association mapping of flowering times with a $60 \mathrm{~K}$ SNP array for this core collection. With quality control and filtration, 20,342 SNP markers were ultimately used for further analyses. In total, 312 SNPs showed marker-trait associations in all four environments, and they were based on a threshold $p$-value of $4.06 \times 10^{-4}$; the 40 QTLs showed significant association with flowering time variations. To explore flowering time QTLs and genes related to growth habits in rapeseed, selection signals related to divergent habits were screened at the genome-wide level and 117 genomic regions were found. Comparing locations of flowering time QTLs and genes with these selection regions revealed that 20 flowering time QTLs and 224 flowering time genes overlapped with 24 and 81 selected regions, respectively. Based on this study, a number of marker-trait associations and candidate genes for flowering time variations in rapeseed were revealed. Moreover, we also showed that both flowering time QTLs and genes play important roles in rapeseed growth habits. These results will be applied to rapeseed breeding programs, and they will aid in our understanding of the relation between flowering time variations and growth habits in plants.

Keywords: growth habits, flowering time, GWAS, rapeseed, B. napus 


\section{INTRODUCTION}

Plants have developed sophisticated systems to adapt to local conditions over the long history of evolution, domestication and natural or artificial selection (Anderson et al., 2011). These systems can be attributed to both physiological and genetic mechanisms, and they have resulted in divergent populations within a single species that grow in different geographic areas or different seasons. This finding further suggests that genomic regions have been targeted by selection and are therefore expected to exhibit characteristic changes in their levels and/or patterns of nucleotide diversity within species. Therefore, insight into these genomic targets and the dissection of the genetic variation of the related traits would improve our understanding of plant adaptation.

Of all the characters involved in growth adaptation, the transition from vegetative growth to flowering is the most crucial factor because it ensures that plants produce enough progeny for their sustainable development. Many plants synchronize their flowering to coincide with seasons by monitoring cues such as the temperature and photoperiod (Jung and Müller, 2009; Zuellig et al., 2014). Additionally, crops are adapted to different growth regions such as fall or spring sowing in different regions. These adaptation processes could enable plants or crops to form different growth habits, such as winter and spring types. For example, two types of annuals, namely biennials (winter annuals) and summer annuals were classified within Arabidopsis thaliana (Amasino, 2004). Usually, plants with different growth habits in one species show very different performance in terms of flowering times. Therefore, this finding suggests that the genetic factors that underlie flowering time variations play important roles in plant habits. However, although flowering time has been extensively studied, its contributions to growth habits are not yet understood. Hence, understanding the genome-wide genetic architecture that controls variations in flowering time and growth habits would be a central goal in plant genetics and genomics.

Rapeseed (Brassica napus L., $2 n=4 x=38$; genome AACC) is one of the most important oilseed crops in the world. This plant is a member in the $U$ triangle and close relations to the model plant A. thaliana (Nagaharu, 1935), the genetic control of flowering time and relevant genetic networks of which have been extensively studied. In A. thaliana, several pathways that control the flowering time have been revealed, namely the photoperiod, vernalization, gibberellic acid (GA), autonomous pathway, and thermal clock (Poethig, 2003). With comparative genetic analyses of these pathways between A. thaliana, B. rapa and B. oleracea with those of $B$. napus, many homologs of flowering time genes have been isolated, and they were shown to be associated with flowering time variations, such as five FLC homologs (BnFLC1 to $B n F L C 5$, and then nine were identified by another group), BnFRI.A3 (FRIGIDA, designated BnaA.FRI.a) and three BnFT paralogs (BnA2.FT, BnC6.FT.a and BnC6.FT.b; Tadege et al., 2001; Wang et al., 2009, 2011; Zou et al., 2012). A number of studies also uncovered the genetic basis of flowering time variations in rapeseed and its relatives through linkage-based QTL mapping. Raman and co-authors reported flowering time as a complex trait in a doubled haploid $(\mathrm{DH})$ rapeseed mapping population that is controlled by at least 20 loci, and it is localized on 10 different chromosomes. These loci each accounted for between 2.4 and $28.6 \%$ of the total genotypic variation for the first flowering and response to vernalization (Van Inghelandt et al., 2012; Raman et al., 2013). Another thorough study was also performed on the basis of a DH population and its derived reconstructed $F_{2}$ population, which was planted in 11 field environments. Many significant-level QTLs (SL-QTL) and micro-real flowering time QTLs (MR-QTL) were detected in each environment (Long et al., 2007; Shi et al., 2009). In addition to these typical reports, there were also other studies that were aimed at dissecting the genetic architecture of flowering time variation in rapeseed and its relatives (Ferreira et al., 1995; Robert et al., 1998; Lou et al., 2007, 2011; Cai et al., 2008; Li et al., 2009; Wang et al., 2011; Hou et al., 2012; Uptmoor et al., 2012; Wu et al., 2012; Raman et al., 2013). However, all this research was usually performed with limited genomic information or small numbers of plant samples.

Genome-wide association mapping (GWAS, also known as GWA) was first used in human disease genetics, and it is primarily based on linkage disequilibrium (LD) in chromosomes (Rannala, 2001). Given that large numbers of plants with a wide genetic range and huge amounts of genomic information can be employed in GWAS, this type of study has attracted more and more attention within plant genomics and genetics (Bradbury et al., 2007; Glaubitz et al., 2014). For rapeseed, there were several case studies of some traits by GWAS of some traits. Cai et al. (2014) performed an association mapping of six yield-related traits in 192 inbred rapeseed lines with 674 molecular markers for genotype data (Cai et al., 2014). For the development of the genotyping technique, an SNP array with $\sim 60 \mathrm{~K}$ SNP markers was produced by an international Brassica consortium. The genetic architecture of seed weights and seed quality was investigated through a genome-wide association study in a large rapeseed core collection panel with this SNP array (Li et al., 2014). Many known and novel significant marker-trait associations were discovered. In addition, another two typical studies were also performed using this $60 \mathrm{~K}$ SNP array in rapeseed. A diversity panel comprising 523 B. napus cultivars and inbred lines were genotyped with this SNP array and a genome-wide association study of flowering time was conducted. Totally, 41 SNPs distributed on 14 chromosomes were found to be associated with flowering time (Xu et al., 2016). Schiessl et al. (2015) identified 101 genome regions associating with the onset of flowering, 69 with plant height, 36 with seed yield and 68 crosstrait regions with potential adaptive value using 158 European winter-type B. napus inbred lines which were also genotyped using this $60 \mathrm{~K}$ SNP array (Schiessl et al., 2015).

In this study, we first performed a genome wide association mapping of flowering time by using the $60 \mathrm{~K} \mathrm{SNP}$ array with a rapeseed core collection panel that contained 448 inbred lines. At least 40 QTLs showed significant association with trait variation. To understand the contributions of flowering time QTLs and genes to growth habits, selection signals corresponding to genomic regions that were related to divergent growth habits were screened in the rapeseed genome. A comparison of the flowering time QTLs and genes with these genomic regions 
revealed that both QTLs and genes play important roles in rapeseed growth habits. According to the performance shown by this study, we were able to provide a novel body of information for understanding the relationship between flowering time variations and growth habits in plants.

\section{MATERIALS AND METHODS}

\section{Plant Materials, Growing Conditions, and Trait Evaluation}

A rapeseed core collection that was reported previously and included 448 inbred lines was used for plant material in this study (Li et al., 2014; Wang et al., 2014). All the pertinent information is listed in Table S1. More detailed information on these inbred lines can be queried through the Chinese Crop Germplasm Resources Information System (CGRIS: http://icgr.caas.net.cn/cgris_english.html). A request for accessions of interest can also be made through CGRIS or by communicating with the corresponding authors. The association panel was grown with 1-3 replications in the field in 2007, 2012 and 2013 (there was one replication in Wuhan in 2013, two replications in Yangluo in 2013 and three replications in Jiangxi at 2007 and 2012). Each accession was grown in a plot with five rows, and each row had 15 plants. The experiment was arranged as a randomized complete block design. In 2007 and 2012, the association panel was grown in the countryside of Jiangxi province (116.27 E, 28.37 N) and seeds were sown on October 1st and September 28th, respectively; in 2013, the association panel was grown in Wuhan (113.68 E, $30.58 \mathrm{~N}$ ) and Yangluo (114.50 E, $30.38 \mathrm{~N}$ ) in Hubei province and seeds were sown on Sep. 28th at both locations. All these places are situated along the Yangtze River, and they are classified as semi-winter growth environments for rapeseed (Wang et al., 2011; Hou et al., 2012; Cai et al., 2014). The agronomic practices were kept uniform in these four growth environments. The flowering times for the in field trials were recorded as the days from sowing to the date when the first flower had opened in $25 \%$ of the plants in each plot. To assign trait data for the four different growth environments, the flowering time corresponding to 2007 and 2012 in Jiangxi province and 2013 in Wuhan and Yangluo in Hubei province were referred to as JX2007, JX2012, WH2013, and YL2013, respectively.

\section{SNP Development and Genotyping}

DNA that had been used for SNP genotyping was isolated from the young leaves of $\sim 10$ plants (pooled) for each of the 448 accessions (plant material was harvested from accessions grown in Wuhan 2013), according to a traditional large-scale CTAB method (Richards et al., 2001). Protocols for SNP genotyping, filtering and location were similar to those of (Li et al., 2014) and they were derived as part of a recently completed study (Wang et al., 2014). In brief, the Brassica $60 \mathrm{~K}$ Illumina ${ }^{\circledR}$ Infinium SNP array was used to obtain genotype data according to the manufacturer's protocols. SNP data were clustered and called up automatically with the Illumina BeadStudio genotyping software. Approximately 25,000 SNPs that did not show three clearly defined clusters that represent the three possible genotypes (AA, $A B$, and $B B$ ) were excluded. The probe sequences of each SNP were used to BLAST against $B$. napus genome sequence V4.1 (Chalhoub et al., 2014). The location of each SNP on 19 B. napus chromosomes was obtained by extracting the top hit positions of its corresponding probe sequence on the genome. Finally, SNPs that showed a minor allele frequency (MAF) of less than 0.05 were excluded from further analyses.

\section{Data Analysis}

Broad sense heritability was calculated as:

$$
H_{B}^{2}=\sigma_{g}^{2} /\left(\sigma_{g}^{2}+\sigma_{g e}^{2} / n+\sigma^{2} / n b\right)
$$

where $\sigma_{g}^{2}$ is the genetic variance, $\sigma_{g e}^{2}$ is the interaction variance between genotypes and environments, $\sigma^{2}$ is the error variance, $\mathrm{n}$ is the number of environments, and $b$ is the number of replications in each experiment. This calculation is similar to Shi et al. (2015). Variance analysis was performed using "aov" function in R software and the formula was set as:

$$
\text { Aov. Fti }=\operatorname{aov}(\mathrm{Fti} \sim \text { Env }+ \text { Gen }+ \text { Envi:Gene, data }=\text { FT })
$$

"FT" indicates the imported data frame of original flowering time data, "Fti" indicates the total variance, "Env" indicates variance produced by environments, "Gen" indicates variance produced by genotypes and "Env:Gen" indicates interaction variance between environments and genotypes. In the variance analysis model, variations of genotypes, environments and interaction between genotypes and environments were considered as fixed effects.

Fixed effects in GWAS models were calculated with a Q or principal component (PCA) matrix, and random effects were calculated with a Kinship (K) matrix. SNPs with MAFs larger than 0.05 and with a distance to adjacent SNPs larger than the mean distance of all 20,342 SNPs were selected for Q and $\mathrm{K}$ matrix analyses. The $\mathrm{Q}$ matrix was predicted with the STRUCTURE v2.3.4 software package (Evanno et al., 2005). The number of groups/sub-populations (k) was set from 1 to 10 with the burn-in period, and the number of MCMC (Markov Chain Monte Carlo) replications after burn-in were both set to 100,000 under the "admixture mode." Five independent runs were performed for each $\mathrm{k}$ number. The $\Delta \mathrm{k}$ method described by Evanno et al. (2005) was used to determine the most likely number of groups/subpopulations. The PCA matrix was calculated by using all the SNPs that were identified by the GCTA tool (Yang et al., 2011), and the first five PCAs were used to construct the PCA matrix.

Six different models were employed for marker-trait associations in this study. In brief, these models were as follows: (1) the naïve-without control for fixed and random effects; (2) the Q model, which controls the fixed effect as the Q matrix; (3) the PCA model, which controls the fixed effects as the PCA matrix; (4) the K model, which controls the random effects as the K matrix; (5) the Q+K model, which controls fixed effects as the Q matrix and the random effects as the K matrix; and (6) the $\mathrm{Q}+\mathrm{K}$ model, which controls the fixed effects as the PCA matrix and the random effects as the $\mathrm{K}$ matrix. The naïve, $\mathrm{Q}$ and PCA models were performed with the GLM algorithm, and the K, 
$\mathrm{Q}+\mathrm{K}$, and $\mathrm{PCA}+\mathrm{K}$ models were run with the MLM algorithm implemented in the TASSEL 4.0 software (Glaubitz et al., 2014).

To identify true marker-trait associations, we applied two criteria to filter the signals produced by the selected association model. First, the $p$-values for all SNPs across all four environments were analyzed by Bonferroni multiple test to calculate the corresponding expected proportion of false discovery rates (FDR) with the "p.adjust" package in the $\mathrm{R}$ program (Benjamini and Hochberg, 1995). A true marker-trait association should show an FDR of less than 0.10. Second, a locus that is thought to have a marker-trait association should harbor at least two SNPs with $p$-values above the first criterion in a 1.5 $\mathrm{Mb}$ region or only one SNP showed marker-trait associations in at least two environments. We then identified candidate genes for the 40 flowering time QTL regions. For the identification of flowering time genes in rapeseed, we first collected all candidate flowering time genes in A. thaliana through published literature (Higgins et al., 2010); then the predicted protein sequences of rapeseed genome V4.1 were used as query to blast all A. thaliana flowering time genes, genes showed $E$-value below $1 \mathrm{E}-20$ were considered as rapeseed flowering time genes. For each rapeseed flowering time gene, their nomenclatures and functions were predicted through their top hit in A. thaliana. For further analyses, flowering time QTLs were assigned names by adding the suffix "qFTi" (it represents the QTL of the flowering time), and their locations including the chromosome number and positions ranges together with ":" as conjunctions; e.g., qFTi:A01:42735035434402 represents the flowering time QTL that is located from 4273503 to 5434402 bp of chromosome A01.

To identify loci that contribute to rapeseed adaptation, an $F_{S T}$ outlier detection method was used to detect the divergence between spring and winter rapeseed. This method was similar to that of a previous study with a slight improvement (Wang et al., 2014). In brief, an artificial selection between spring and winter rapeseed sub-populations was estimated on the basis of the pure drift model (Nicholson et al., 2002), by following the procedure as previously described. Genomic regions that were subjected to the above artificial selection were regarded as the primary factors that contributed to growth adaptation. The $F_{S T}$ values were calculated for each SNP first, and then the average $F_{S T}$ within a $0.5 \mathrm{Mb}$ window with a $50 \mathrm{~kb}$ sliding bin for each of the 19 chromosomes was calculated. The mean $F_{S T}$ was set at zero when the $0.5 \mathrm{Mb}$ windows contained fewer than 2 SNP markers. Subsequently, the genomic regions ( $0.5 \mathrm{Mb}$ windows) showing with $F_{S T}$ value above the 10th percentile were regarded as the targets of artificial selection. For the classification of the growth types, the types were carefully collated and curated on the basis of their place of origin, recorded pedigrees, breeding records and previous field performance.

\section{RESULTS}

\section{Flowering Time Variations in a Rapeseed Core Collection Panel}

According to the trait evaluation that was performed in the fields, extensive flowering time variation was observed between or within environments (Table 1 and Figure 1). Figure 1 and Table 1 showed flowering times ranging from 155 to 177 days in environment JX2007, 77 to 182 days in environment JX2012, 75 to 181 days in environment YL2013 and 115 to 164 days in environment WH2013. In comparing the flowering times across environments, although the correlation between pairs of environments showed in a close level, large variations were also clearly observed (Table 2 and Figure 2). YL2013 showed the longest average flowering time at $164.8 \pm 12.8$ days, whereas WH2013 showed the shortest with $154.6 \pm 8.6$ days (Table 1). These data indicate that the flowering time for rapeseed could be greatly affected by environment. To test the effects of genotypes, environments and their interactions on flowering time variation, a variance analysis was conducted on our core collection panel. Table 1 reveals that the genotype $(G)$, environment (E) and genotype-by-environment interaction $(\mathrm{G} \times \mathrm{E})$ have significant effects on flowering time variation $(p<0.01)$. According to this calculation, the broad sense heritability of the flowering time is $\sim 83 \%$ (Table $\mathbf{1}$ ).

\section{Evaluation of Association Mapping Models}

To create a Q matrix for all 448 rapeseed accessions, the $\Delta \mathrm{k}$ method was used to determine the most likely number of groups/sub-populations. The results showed that $k=2$ produced the highest $\Delta \mathrm{k}$ value, and therefore, it was selected to create the $\mathrm{Q}$ matrix, This result was similar to that of (Wang et al., 2014). To assess the utility of each GWAS model, observed $p$-values [observed $-\log _{10}$ ( $p$-value)] across all four environments from each model and their expected ranked values [expected $-\log _{10}(p$-value $\left.)\right]$ were plotted in a quantile-quantile (QQ) figure (Figure 3). An ideal model should show uniformity between the observed and expected $p$-values in the QQ plot. According to Figure 3, the Q+K, PCA $+\mathrm{K}, \mathrm{K}$ and PCA models showed much better correlation than naïve and Q models. Slight

TABLE 1 | Analysis of variance for rapeseed flowering time.

\begin{tabular}{|c|c|c|c|c|c|}
\hline \multicolumn{6}{|c|}{ Flowering time data } \\
\hline & Mean & $S D$ & CV (\%) & Min. & Max. \\
\hline JX2007 & 163.4 & 4.5 & 2.8 & 155.0 & 177.0 \\
\hline JX2012 & 159.8 & 12.9 & 8.1 & 77.0 & 182.0 \\
\hline WH2O13 & 154.6 & 8.6 & 5.6 & 115.0 & 164.0 \\
\hline YL2013 & 164.8 & 12.8 & 7.8 & 75.0 & 181.0 \\
\hline \multicolumn{6}{|c|}{ Variance analysis } \\
\hline Source & DF & ss & MS & $F$-value & $\operatorname{Pr}(>F)$ \\
\hline G (genotype) & 447 & 241,128 & 539 & 44 & $<2 \mathrm{E}-16$ \\
\hline E (environment) & 3 & 106,783 & 35594 & 2900 & $<2 \mathrm{E}-16$ \\
\hline$G \times E$ & 1172 & 126,352 & 108 & 8.8 & $<2 \mathrm{E}-16$ \\
\hline Residuals & 2062 & 25,306 & 12.3 & & \\
\hline
\end{tabular}

$S D$ indicates the standard deviation of the flowering time. CV indicates the coefficient of variation. The unit for the mean, SD, Min. and Max. is days. DF indicates the degrees of freedom, SS indicates the sum of squares, MS indicates the mean square. 
JX2007
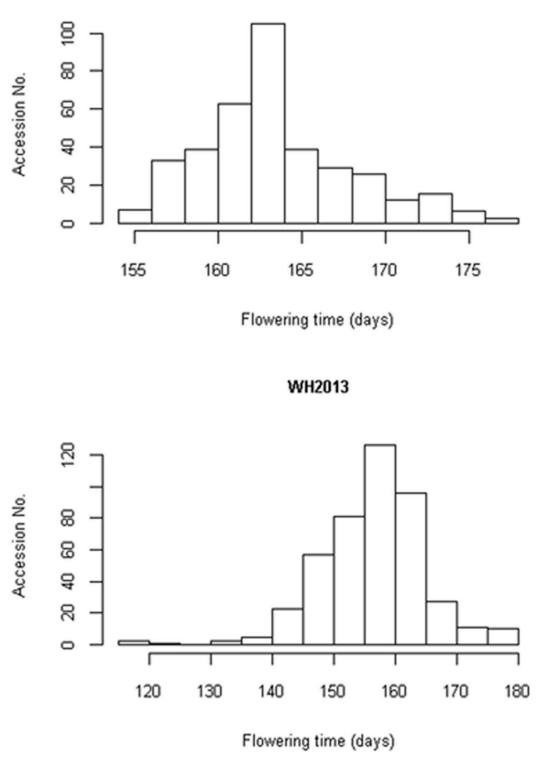

JX2012
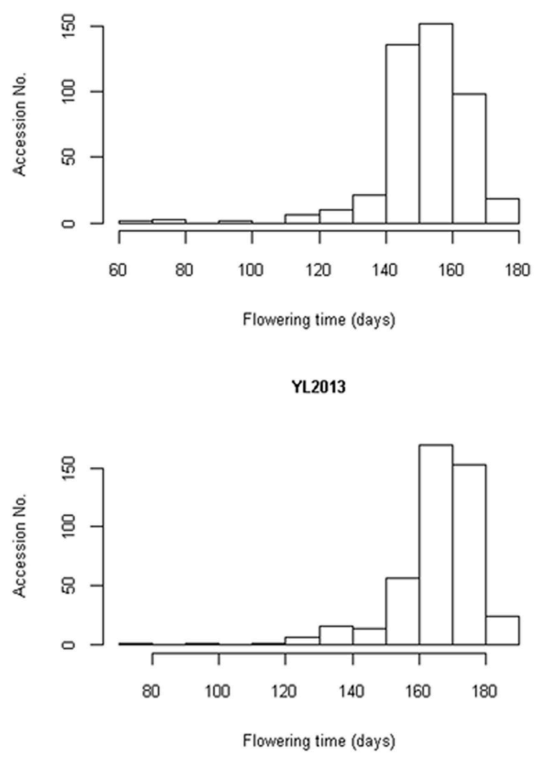

FIGURE 1 | Distribution of flowering time in four different environments. The rapeseed core collection panel of 448 lines was planted in four different environments for 3 years, namely 2007, 2012, and 2013. In 2007 and 2012, the panel was grown in Jiangxi, and the codes for these two environments are JX2007 and JX2012, respectively. In 2013, the panel was grown in Wuhan and Yangluo, and the codes for these two environments are WH2013 and YL2013, respectively. The $X$ axis indicates the flowering time (days) and the $Y$ axis indicates accession number.

differences were observed among the $\mathrm{Q}+\mathrm{K}, \mathrm{PCA}+\mathrm{K}, \mathrm{K}$ and $\mathrm{PCA}$ models. However, the $\mathrm{Q}+\mathrm{K}$ model was somewhat better than the others, therefore, this model was selected to detect marker-trait associations.

\section{Genome-Wide Association Studies and Candidate Gene Identification}

By using Bonferroni multiple testing to control the false discovery rate in the $\mathrm{Q}+\mathrm{K}$ association model (Benjamini and Hochberg, 1995), a $p$-value of $4.06 \times 10^{-4}\left[-\log _{10}(p\right.$-value $\left.)=3.39\right]$ revealed an FDR of 0.10 . This criterion is usually selected as the threshold in GWAS studies (Cai et al., 2014). Therefore, SNPs with $p$-values of less than $4.06 \times 10^{-4}$ were considered candidates for flowering marker-trait association. In total, 312 SNPs showed marker-trait associations in all four environments based on the threshold. Because Wang et al. reported that the distance between SNPs approached $1.5 \mathrm{Mb}$, the average $r^{2}$ was 0.1 (Wang et al., 2014). This LD level usually indicates that there is nearly no linkage between the markers; thus, we defined SNPs within a $1.5 \mathrm{Mb}$ window as a single locus. The 312 pairs of markertrait association SNPs could be merged into 40 loci (Table S2 and Figure 4). Twenty-two SNPs could not be assigned into any marker-trait association loci because they did not meet the criterion of at least two SNPs with $p$-values above the threshold in a $1.5 \mathrm{Mb}$ region. According to these data, we can conclude that we have identified 40 flowering time QTLs by genome-wide association mapping in the rapeseed core collection from four environments.

Of these 40 flowering time QTLs, 19 could be detected in at least two environments and they are located on chromosomes
TABLE 2 | Flowering time correlation coefficients between pairs of environments.

\begin{tabular}{llll}
\hline & JX2012 & WH2013 & YL2013 \\
\hline JX2007 & $0.428^{* \star *}$ & $0.631^{\star \star *}$ & $0.526^{\star \star \star}$ \\
JX2012 & & $0.702^{\star \star *}$ & $0.730^{\star \star *}$ \\
WH2013 & & $0.850^{\star \star *}$
\end{tabular}

*** indicates a p-value of less than 0.001 .

A01, A02, A03, A04, A06, A07, A10, C02, C03, C04, and C09 (Figure 4 and Table 3). Four QTLs, namely qFTi:A04:2570404734286, qFTi:A07:14463578-18554138, qFTi:A10:1337510415191366 and qFTi:C02:6956919-13653054 could be detected in all four environments. Two could be detected in three environments, namely qFTi:A01:9984823-11863045 and qFTi:A01:13863759-15685448. qFTi:C02:6956919-13653054 showed the highest probability of marker-trait association with $-\log _{10}$ ( $p$-values) equal to 6.5 , and it showed the largest contribution rate to flowering time variance with $R^{2}$ equal to $\sim 8.77 \%$.

Flowering time genes in A. thaliana were obtained through published literature (Higgins et al., 2010). Genes that were involved in flowering time pathways in rapeseed were obtained according to close relation between $A$. thaliana and the Brassica genus (see Materials and Methods).Totally, 1520 candidate flowering time genes were identified in the newly released $B$. napus genome. All candidate flowering time genes and their corresponding locations on rapeseed chromosomes are listed in Table S3. By comparing the locations of flowering 
JX2007 vs. JX2012

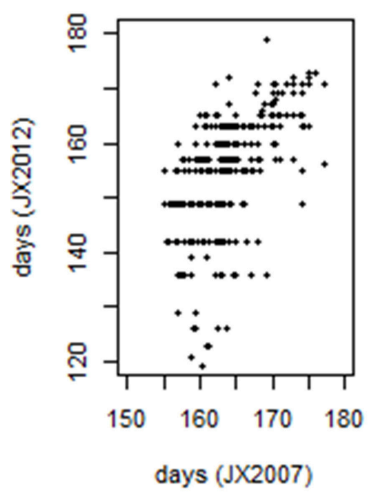

JX2012 vs. WH2013

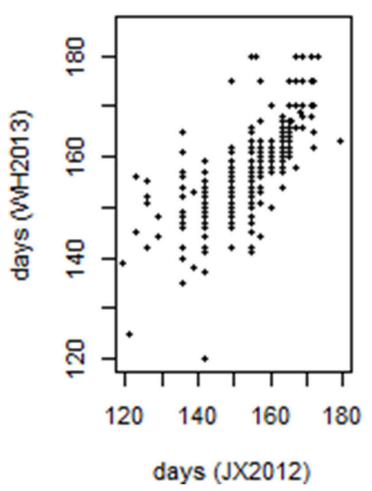

JX2007 vs. WH2013

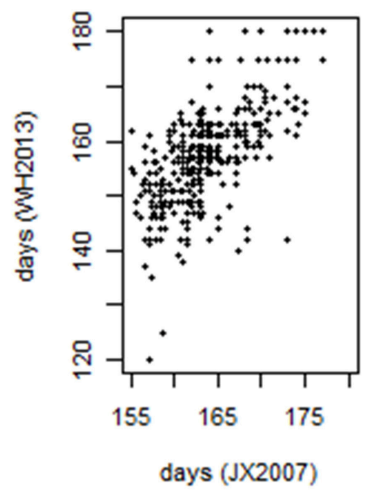

JX2012 vs. YL2013

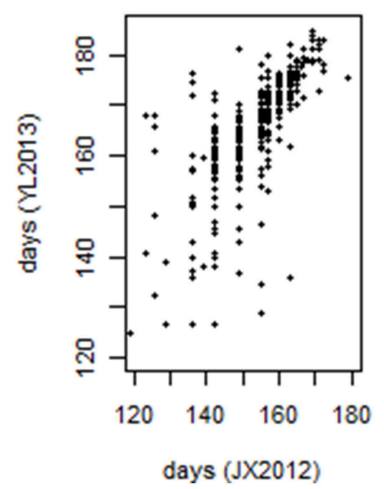

JX2007 vs. YL2013

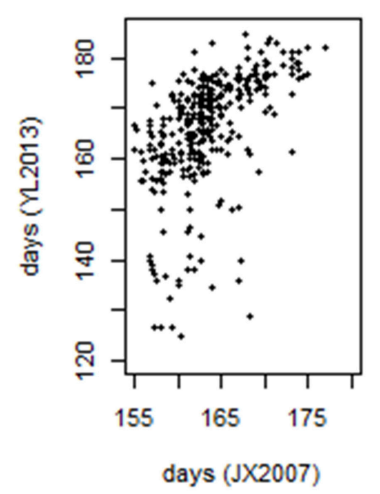

WH2013 vs. YL2013

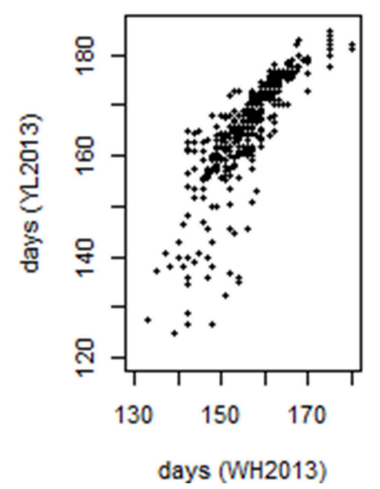

FIGURE 2 | Pair-plots of flowering time correlation coefficients.

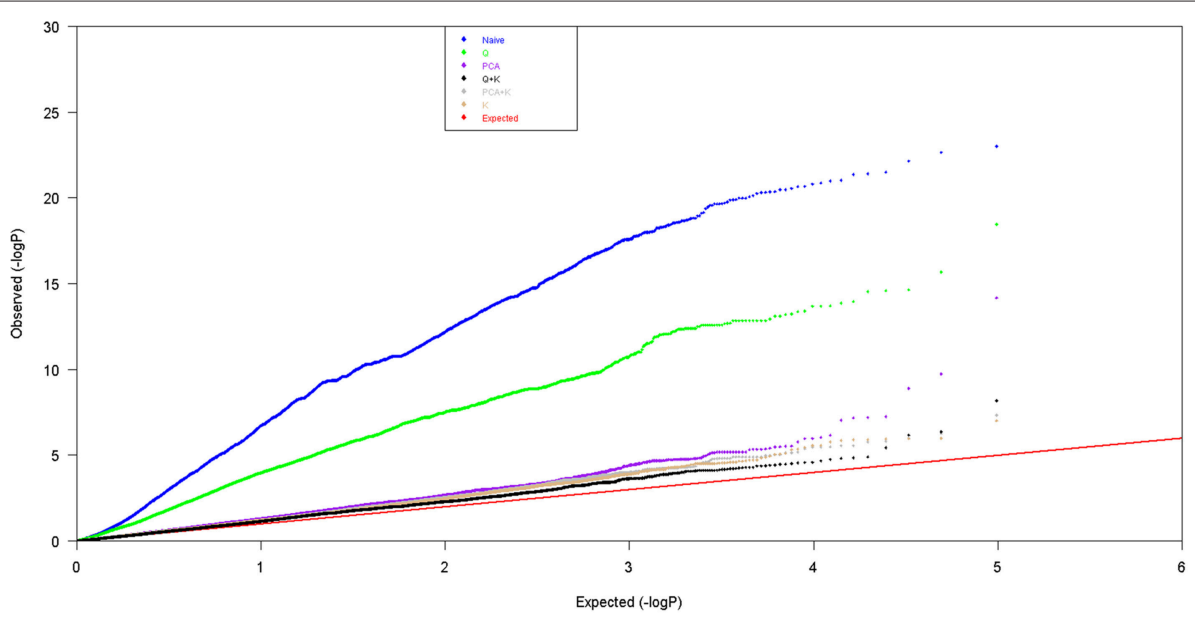

FIGURE 3 | QQ plots of the estimated - $\log _{10}(p$-value) from association analyses using six different models.

time QTLs with flowering time candidate genes on rapeseed chromosomes, we were able to identify candidate genes for 25 of the 40 flowering time QTLs (Table S2). For these 25 QTLs, 19 showed more than one candidate gene in a locus. For example, there are 12 flowering time genes located within qFTi:A04:257040-4734286; they are CDF3, AGL24, CKA2, AGL24, CIB1, AGL18, PIE1, AREB3, CDF2, AGL32, CKA2, and ARP6. 

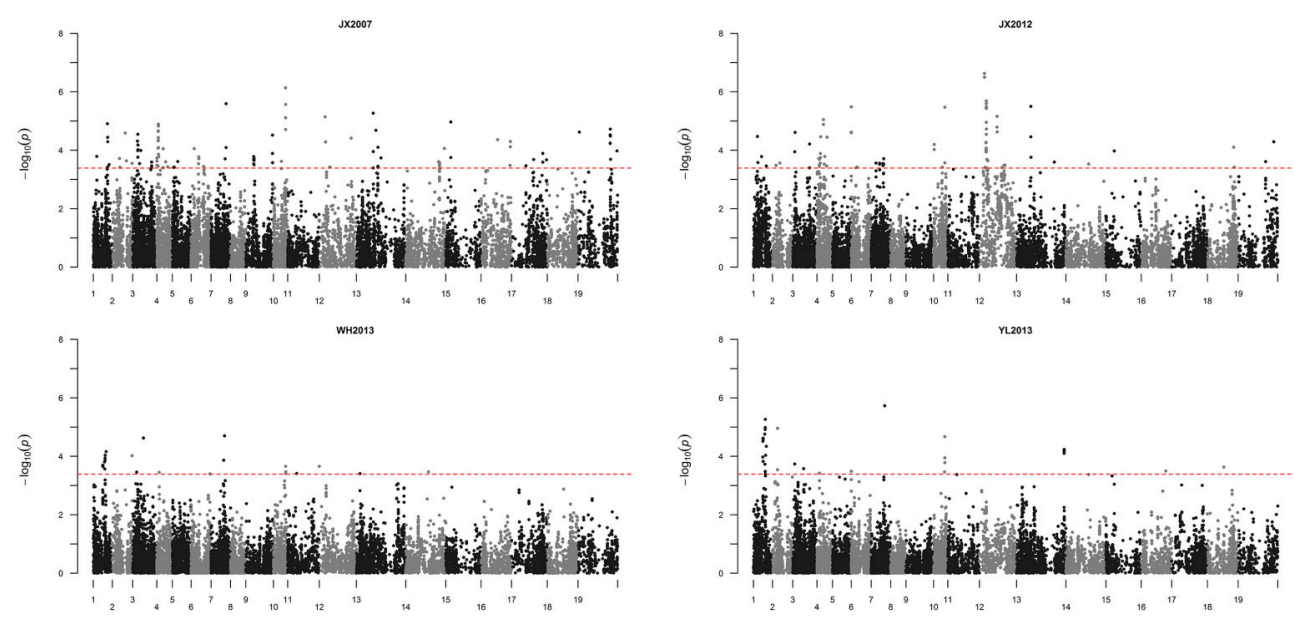

FIGURE 4 | Manhattan plots of association analysis using the Q+K model for rapeseed flowering times in four environments. The X-axis indicates the SNP positions on 19 chromosomes within the rapeseed genome. They were arranged from A01 to A10 and C01 to C09 with alternating color plots of black and gray. Numbers 1 to 10 correspond to chromosomes A01 to A10, and numbers 11 to 19 correspond to chromosomes C01 to C09. The red dashed line in each environment indicates that the threshold $p$-value is equal to $3.83 \times 10^{-4}$. The $X$-axis indicates the $-\log _{10}(p$-value) for each SNP.

TABLE 3 | Marker-trait associations were detected in at least two environments for rapeseed flowering times.

\begin{tabular}{|c|c|c|c|c|c|}
\hline \multirow{2}{*}{$\begin{array}{l}\text { Chr. } \\
A 01\end{array}$} & \multirow{2}{*}{$\frac{-\log 10(P)^{a}}{4.47}$} & \multirow{2}{*}{$\begin{array}{c}\text { Max R2 (\%) } \\
4.73\end{array}$} & \multicolumn{2}{|c|}{ Range $(\mathbf{b p})^{\mathbf{b}}$} & \multirow{2}{*}{$\begin{array}{l}\text { Environments }^{c} \\
J X 2007, J X 2012\end{array}$} \\
\hline & & & $4,273,503$ & $5,434,402$ & \\
\hline $\mathrm{A} 01$ & 4.62 & 4.89 & $9,984,823$ & $11,863,045$ & JX2012, WH2013, YL2013 \\
\hline $\mathrm{A} 02$ & 3.72 & 4.56 & $8,776,742$ & $9,248,051$ & JX2007, JX2012 \\
\hline A02 & 3.55 & 4.33 & $24,150,742$ & $24,237,453$ & JX2007, WH2013 \\
\hline A03 & 4.63 & 4.86 & $13,297,841$ & $13,297,841$ & WH2013, YL2013 \\
\hline A04 & 4.89 & 6.00 & 257,040 & $4,734,286$ & JX2007, JX2012, WH2013,YL2013 \\
\hline A04 & 5.05 & 5.81 & $7,743,947$ & $10,942,653$ & JX2007, JX2012 \\
\hline A04 & 4.45 & 4.70 & $11,898,475$ & $13,460,703$ & JX2007, JX2012 \\
\hline A06 & 4.62 & 4.93 & 42,625 & 73,593 & JX2012, YL2013 \\
\hline $\mathrm{C} 02$ & 6.50 & 8.77 & $6,956,919$ & $13,653,054$ & JX2007, JX2012, WH2013, YL2013 \\
\hline $\mathrm{CO3}$ & 5.27 & 6.53 & $17,612,972$ & $20,733,429$ & JX2007, HX2012 \\
\hline C04 & 3.53 & 3.71 & $27,699,177$ & $27,699,177$ & JX2012, WH2013 \\
\hline Co9 & 4.72 & 5.79 & $39,312,343$ & $43,429,210$ & JX2007, JX2012 \\
\hline
\end{tabular}

${ }^{a}-\log P$ is the $-\log _{10}$ ( $p$-value) for the lead SNPS.

${ }^{b}$ The locations of SNPS show the -logP above the criterion of 3.42 .

${ }^{c}$ Codes are identical with those of Figure 1.

In the QTL regions with candidate genes, we found that some were similar to those identified in previous studies. Two candidate genes within qFTi:A03:5046910-6515058, namely FRI and $F L C$, were also reported by two previous studies (Wang et al., 2011; Zou et al., 2012). In using an important flowering time gene called, $F L C$, as a common anchored marker, we found that qFTi:A10:13375104-15191366 was also reported by previous flowering time QTL mapping using a DH population (Long et al., 2007; Wang et al., 2011; Hou et al., 2012; Zou et al., 2012). The FLC gene in this locus that is involved in the vernalization pathway is located at $14,998,617$ to $15,003,197 \mathrm{bp}$; it is only $\sim 145$ $\mathrm{kb}$ from the lead SNP (SNPs showed the largest $p$-value and $r^{2}$ for this QTL), Bn-A10-p14914898 (Tables S2, S3). Additionally, as information is obtained, some flowering time QTLs would be 
novel, such as qFTi:A01:4273503-5434402 and qFTi:A04:2570404734286. According to these results, we can conclude that a number of loci and genes explain flowering time variations in the core collection.

\section{Contributions of Flowering Time QTLs and Genes to Rapeseed Growth Habits}

To investigate the contributions of flowering time QTLs and genes to rapeseed growth habits, we first identified the influence of artificial selection in the formation of different growth types in rapeseed. The growth type for each accession in our core collection panel is listed in Table S1. Because it was difficult to determine the exact growth type characteristics for semi-winter rapeseed, this growth type was excluded from the analysis. There were 106 spring and 153 winter rapeseed growth types (Table S1). Because $F_{S T}$ is not strongly affected by ascertainment bias, it is better suited for analyzing data that are generated by SNP chips (Albrechtsen et al., 2010). We performed a genome-wide scan for selection signatures in our set of 259 B. napus accessions including spring and winter rapeseed growth types by estimating Wright's $F_{S T}$ with a sliding window method (see Materials and Methods), and the 10th percentile $F_{S T \text {-window was said to }}$ include loci that were subjected to artificial selection, which was responsible for the divergence of rapeseed adaptation. A total of 117 loci equal to $124.0 \mathrm{Mb}$ of the genome met our criterion. This finding indicated that nearly one-tenth of the genome (the total size for the whole rapeseed genome is approximately $1.2 \mathrm{~Gb}$ ) contributed to the divergence that occurred between spring and winter growth habits for rapeseed. These 117 loci were simply named "selection regions." We then compared these 117 regions on 19 chromosomes with all 40 flowering time loci. Twenty flowering time QTLs overlapped with 24 selection regions (Figure 5 and Table S4). Most of the 20 QTLs made large contributions to the flowering time variation and could be detected in more than one environment in an association mapping study (Table 3 and Table S2). When comparing the locations of flowering time candidate genes in the rapeseed genome within these selection regions, 224 out of 1520 flowering time genes fell into these 81 selection regions (Figure 5 and Table S4). Based on an investigation of annotation information for these 224 genes, most are involved in flowering time regulation through pathways for the photoperiod, vernalization, GA, autonomous pathway, and thermal clock (Table S3).

\section{DISCUSSION}

In this study, we were able to detect 40 flowering time QTLs by genome-wide association analysis by using a large rapeseed core collection panel. However, we did not know which of these QTLs had already been reported and which were new. Therefore, comparisons of our results with previous studies by anchoring a known marker sequence to rapeseed chromosomes would answer this question. Because the information from many for lots of studies was not complete, we only compared our results with TN DH mapping population and another two similar studies which also used $60 \mathrm{~K} \mathrm{SNP}$ array to perform genotyping. Totally, we found that 7 of the 40 flowering QTLs in this study have been reported by studies on TN DH mapping population, 5 have been reported by studies performed on 158 European wintertype B. napus inbred lines (Schiessl et al., 2015) and 5 have been reported by studies performed on a diversity panel comprising 523 B. napus cultivars and inbred lines (Xu et al., 2016) (Table S2). In sum, 13 of the 40 flowering QTLs in this study can be found in at least one of other studies which were used in comparison. Given the limited information and different algorithms between linkage (QTL mapping) and linkage disequilibrium mapping (GWAS), we speculated that the numbers of QTLs reported by previous studies would be larger than 13 . Of these 13 common QTLs, some of them were reported in many environments, such as qFTi:A10:13375104-15191366 and qFTi:C02:695691913653054, which indicated that these loci could control the flowering times under different growth conditions, and they were very stable. For further insights into these novel QTLs in Table S2, they tended to have no candidate genes. This finding suggested that further studies should focus on these QTLs and it also revealed the powerful nature of the GWAS when used with our core collection panel. Moreover, in the association mapping analysis, some QTLs showed very high contributions to flowering time variations, such as qFTi:C02:6956919-13653054, which explained $\sim 9 \%$ of the variance (Table 3 and Table S2). Because the SNP markers used in this study are also publicly available in the Brassica Consortium, the information on markertrait associations and candidate flowering time genes can guide marker assistant selection (MAS) in rapeseed breeding programs to come. Therefore, based on all the results from this study, we can conclude that we have provided very valuable data and resources for further rapeseed research.

In this study, we evaluated the flowering time data from four independent environments over three different years. Large variations across different environments were shown in Figure 1, and thus, this panel should be highly suitable for a GWAS study. Moreover, strong interactions between genotypes and environments were also revealed. This finding indicates that variations in environments make large contributions to flowering time variations. In addition, when comparing our results with previous flowering time studies for rapeseed, many of the flowering QTLs reported by others were not detected in this study. For example, QTLs on linkage groups C09 (Luo et al., 2014) and A05 (Long et al., 2007) could not be identified in the present study. This omission may be attributed to the fact that we only grew the rapeseed core collection panel in four environments: JX2007, JX2012, WH2013, and YL2013. Though they could be assigned to different places or different growing years, all of them were still located in central China along the Yangtze River. Therefore, the diversity of climate factors, such as the temperature, photoperiod and humidity, within these four environments is not sufficient to uncover all the mechanisms underlying the flowering time variation. This finding suggests that we should grow this collection panel in more environments to uncover more QTLs that are associated with the flowering time variation.

During further analysis of the flowering time QTL regions and their candidate genes (Table S2), it was common to find more 


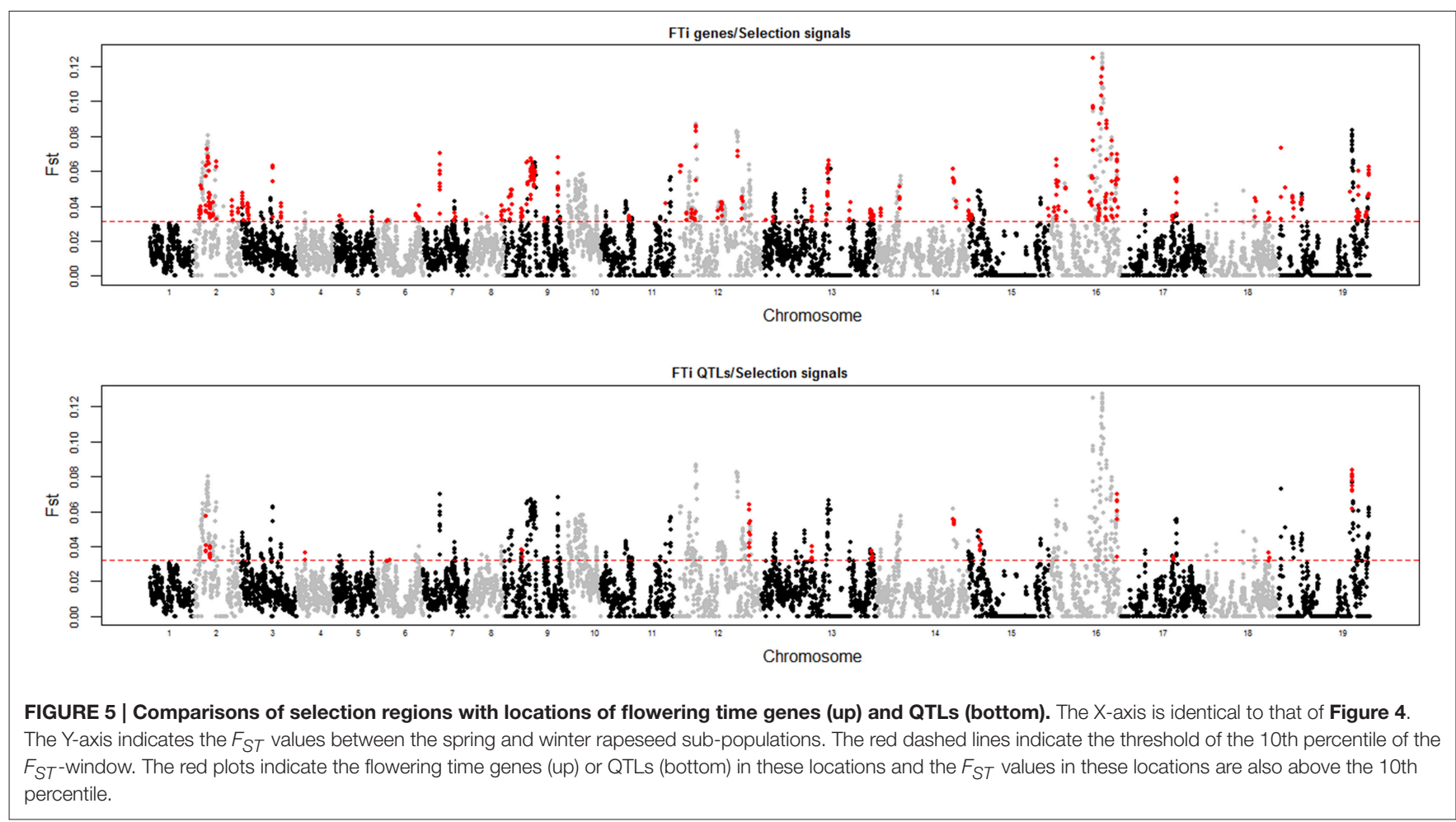

than one candidate flowering time gene within a single QTL. This result suggests some possible patterns in the rapeseed genome and in flowering time regulation. First, genes that perform functions in the same or related pathways tend to be located in clusters in the genome. Similar discoveries were also reported in a number of other studies (e.g., Lee and Sonnhammer, 2003; Lee and Chang, 2013). This phenomenon would be more likely because rapeseed is an allotetraploid plant, and the two progenitors, that is, B. rapa and B. oleracea, also have a close genetic relationship. Second, different flowering time genes that are clustered in the same genomic region in rapeseed would have experienced co-evolution or co-selection. We found divergent signals from $F_{S T}$ between spring and winter rapeseed types. The genomic regions with outlier $F_{S T}$ values were usually the targets of natural or artificial selection. Genetic factors including coding and non-coding elements within those targets in the same subpopulation should have undergone similar selective pressures. Therefore, these elements should show the same patterns of evolution or selection. This assumption is very similar to a previous study on flowering time genes. Zou et al. noted that functionally related flowering time genes are tightly linked in Brassica species, and these tightly linked flowering time genes exhibited co-expression patterns (Zou et al., 2012). However, the mechanism for underlying the flowering time gene clustering in the rapeseed genome is still not clear, and more studies should be performed on this subject.

According to the investigation of genomic regions that corresponded to growth habits in rapeseed, 117 loci equal to $124 \mathrm{Mb}$ of the genome showed possible selection signals (Table $\mathrm{S} 4$ ). Given that the total size of genome is $\sim 1.2 \mathrm{~Gb}$, this finding suggests that $10 \%$ of the genome would be the target of selective pressures during the growth habit-shaping processes in rapeseed. There is only a $\sim 7500-10,000$ year history for this plant and as a result of slow linkage disequilibrium decay in the genome (Nagaharu, 1935; Qian et al., 2014; Wang et al., 2014), many genetic elements located in the selection regions would have no direct effects on growth adaptation but would only be detected by "linkage-drag." For insight into the procedures used to screen of the selection regions in this study, a low stringent threshold for an $F_{S T}$ above the 10th percentile value was employed. This threshold would overestimate the selection signals in the whole genome. However, because the transition from vegetative growth to flowering is one of the crucial direct factors in determining plant growth habits (Anderson et al., 2011), combining the analyses of flowering time QTLs and candidate genes would have high efficiency and accuracy at uncovering the mechanisms that underlie growth adaptation. This result also suggests that the result of QTL or candidate genes findings that were involved in growth habits in this study would be very reliable. Additionally, we found that 20 out of 40 flowering time QTLs overlapped the 24 selected loci that are important in determining rapeseed growth habits (Figure 4 and Table S4). This finding indicates that $~ 50 \%$ QTLs could be involved in this biological process. By contrast, 224 out of 1520 flowering time candidate genes were detected when determining rapeseed growth habits (Figure 4 and Table S4), which is $\sim 15 \%$ of the total flowering time genes. This pattern may indicate that although there are numerous flowering time genes in the rapeseed genome, not all genes perform their full functions. This lack of full activity is very common for duplicated genes in complex genomes (Wang et al., 2013). 
Additionally, although large flowering time variations were observed among the four environments for our 448 inbred lines, these environments for GWAS still belong to the same climate area (semi-winter) along the Yangtze River in central China, and as a result, not all QTLs that were responsible for flowering time variation in our panel were identified. More overlaps would be observed between the selection and flowering time QTLs if the GWAS studies were performed in more divergent environments.

In this study, a wide distribution and large variation of flowering times were observed in the rapeseed core collection. Based on a variance analysis of flowering time data, the wide inheritance of this trait was also observed. Moreover, both the environment and the interactions between environments and genotypes showed significant effects in controlling the flowering time variation. Following genome-wide association mapping, 312 SNPs showed marker-trait associations in all four environments based on the $p$-value threshold of $4.06 \times 10^{-4}$ and 40 QTLs showed a significant association with flowering time variation. These SNPs are distributed on 17 of the 19 chromosomes, with the exceptions of chromosome A08 and C01. These marker-trait associations and flowering time genes can be easily applied to advance rapeseed breeding programs. To further understand the contributions of flowering time QTLs and genes to growth habits, selection signals related to divergent adaptation in rapeseed were scanned at a genome-wide level. A locations comparison of flowering time QTLs and genes with these genomic regions

\section{REFERENCES}

Albrechtsen, A., Nielsen, F. C., and Nielsen, R. (2010). Ascertainment biases in SNP chips affect measures of population divergence. Mol. Biol. Evol. 27, 2534-2547. doi: $10.1093 / \mathrm{molbev} / \mathrm{msq} 148$

Amasino, R. (2004). Vernalization, competence, and the epigenetic memory of winter. Plant Cell 16, 2553-2559. doi: 10.1105/tpc.104.161070

Anderson, J. T., Willis, J. H., and Mitchell-Olds, T. (2011). Evolutionary genetics of plant adaptation. Trends Genet. 27, 258-266. doi: 10.1016/j.tig.2011.0 4.001

Benjamini, Y., and Hochberg, Y. (1995). Controlling the false discovery rate: a practical and powerful approach to multiple testing. J. R. Stat. Soc. Ser. B 57, 289-300.

Bradbury, P. J., Zhang, Z., Kroon, D. E., Casstevens, T. M., Ramdoss, Y., and Buckler, E. S. (2007). TASSEL: software for association mapping of complex traits in diverse samples. Bioinformatics 23, 2633-2635. doi: 10.1093/bioinformatics/btm308

Cai, C. C., Tu, J. X., Fu, T. D., and Chen, B. Y. (2008). The genetic basis of flowering time and photoperiod sensitivity in rapeseed (Brassica napus L.). Genetika 44, 381-388. doi: 10.1134/s1022795408030137

Cai, D., Xiao, Y., Yang, W., Ye, W., Wang, B., Younas, M., et al. (2014). Association mapping of six yieldrelated traits in rapeseed (Brassica napus L.). Theor. Appl. Genet. 127, 85-96. doi: 10.1007/s00122-013-2203-9

Chalhoub, B., Denoeud, F., Liu, S., Parkin, I. A., Tang, H., Wang, X., et al. (2014). Plant genetics. Early allopolyploid evolution in the post-Neolithic Brassica napus oilseed genome. Science 345, 950-953. doi: 10.1126/science.1253435

Evanno, G., Regnaut, S., and Goudet, J. (2005). Detecting the number of clusters of individuals using the software STRUCTURE: a simulation study. Mol. Ecol. 14, 2611-2620. doi: 10.1111/j.1365-294X.2005.02553.x

Ferreira, M. E., Satagopan, J., Yandell, B. S., Williams, P. H., and Osborn, T. C. (1995). Mapping loci controlling vernalization requirement and flowering time in Brassica napus. Theor. Appl. Genet. 90, 727-732. doi: 10.1007/BF002 22140 revealed that both QTLs and genes played important roles in rapeseed growth habits. Based on this study, we have provided very valuable data and resources for further rapeseed research.

\section{AUTHOR CONTRIBUTIONS}

NW and XW organized and supported the entire study. NW, BC, KX, GG, FL, JQ, GY, JL, and HL performed the flowering time evaluation and SNP genotyping. NW also wrote and edited this manuscript.

\section{ACKNOWLEDGMENTS}

This work was supported by the National Science and Technology Pillar Program during the Twelfth Five-year Plan Period (2013BAD01B03 and 2013BAD01B01) and Crop Germplasm Protection Project (NB2011-2130135). The authors are grateful to AJE (American Journal Experts) for improving the English in this paper.

\section{SUPPLEMENTARY MATERIAL}

The Supplementary Material for this article can be found online at: http://journal.frontiersin.org/article/10.3389/fpls.2016. 00338

Glaubitz, J. C., Casstevens, T. M., Lu, F., Harriman, J., Elshire, R. J., Sun, Q., et al. (2014). TASSEL-GBS: a high capacity genotyping by sequencing analysis pipeline. PLoS ONE 9:e90346. doi: 10.1371/journal.pone.0090346

Higgins, J. A., Bailey, P. C., and Laurie, D. A. (2010). Comparative genomics of flowering time pathways using Brachypodium distachyon as a model for the temperate grasses. PLoS ONE 5:e10065. doi: 10.1371/journal.pone. 0010065

Hou, J., Long, Y., Raman, H., Zou, X., Wang, J., Dai, S., et al. (2012). A Tourist-like MITE insertion in the upstream region of the BnFLC.A10 gene is associated with vernalization requirement in rapeseed (Brassica napus L.). BMC Plant Biol. 12:238. doi: 10.1186/1471-2229-12-238

Jung, C., and Müller, A. E. (2009). Flowering time control and applications in plant breeding. Trends Plant Sci. 14, 563-573. doi: 10.1016/j.tplants.2009.07.005

Lee, J. M., and Sonnhammer, E. L. (2003). Genomic gene clustering analysis of pathways in eukaryotes. Genome Res. 13, 875-882. doi: 10.1101/gr.737703

Lee, Y. C., and Chang, H. H. (2013). The evolution and functional significance of nested gene structures in Drosophila melanogaster. Genome Biol. Evol. 5, 1978-1985. doi: 10.1093/gbe/evt149

Li, F., Chen, B., Xu, K., Wu, J., Song, W., Bancroft, I., et al. (2014). Genomewide association study dissects the genetic architecture of seed weight and seed quality in rapeseed (Brassica napus L.). DNA Res. 21, 355-367. doi: 10.1093/dnares/dsu002

Li, F., Kitashiba, H., Inaba, K., and Nishio, T. (2009). A Brassica rapa linkage map of EST-based SNP markers for identification of candidate genes controlling flowering time and leaf morphological traits. DNA Res. 16, 311-323. doi: 10.1093/dnares/dsp020

Long, Y., Shi, J., Qiu, D., Li, R., Zhang, C., Wang, J., et al. (2007). Flowering time quantitative trait Loci analysis of oilseed brassica in multiple environments and genomewide alignment with Arabidopsis. Genetics 177, 2433-2444. doi: 10.1534/genetics.107.080705

Lou, P., Xie, Q., Xu, X., Edwards, C. E., Brock, M. T., Weinig, C., et al. (2011). Genetic architecture of the circadian clock and flowering time in Brassica rapa. Theor. Appl. Genet. 123, 397-409. doi: 10.1007/s00122-011-1592-x 
Lou, P., Zhao, J., Kim, J. S., Shen, S., Del Carpio, D. P., Song, X., et al. (2007). Quantitative trait loci for flowering time and morphological traits in multiple populations of Brassica rapa. J. Exp. Bot. 58, 4005-4016. doi: 10.1093/jxb/erm255

Luo, Y. X., Luo, C. Y., Du, D. Z., Fu, Z., Yao, Y. M., Xu, C. C., et al. (2014). Quantitative trait analysis of flowering time in spring rapeseed (B. napus L.). Euphytica 200, 321-335. doi: 10.1007/s10681-014-1140-2

Nagaharu, U. (1935). Genome analysis in Brassica with special reference to the experimental formation of B. napus and peculiar mode of fertilization. Japan. J. Bot. 7, 389-452.

Nicholson, G., Smith, A. V., Jónsson, F., Gstafsson, Stefánsson, K., and Donnelly, P. (2002). Assessing population differentiation and isolation from singlenucleotide polymorphism data. J. R. Stat. Soc. Ser. B 64, 695-715. doi: 10.1111/1467-9868.00357

Poethig, R. S. (2003). Phase change and the regulation of developmental timing in plants. Science 301, 334-336. doi: 10.1126/science.1085328

Qian, L., Qian, W., and Snowdon, R. J. (2014). Sub-genomic selection patterns as a signature of breeding in the allopolyploid Brassica napus genome. BMC Genomics 15:1170. doi: 10.1186/1471-2164-15-1170

Raman, H., Raman, R., Eckermann, P., Coombes, N., Manoli, S., Zou, X., et al. (2013). Genetic and physical mapping of flowering time loci in canola (Brassica napus L.). Theor. Appl. Genet. 126, 119-132. doi: 10.1007/s00122-012-1 966-8

Rannala, B. (2001). Finding genes influencing susceptibility to complex diseases in the post-genome era. Am. J. Pharmacogenomics 1, 203-221. doi: 10.2165/00129785-200101030-00005

Richards, E., Reichardt, M., and Rogers, S. (2001). Preparation of genomic DNA from plant tissue. Curr. Protoc. Mol. Biol. Chapter 2, Unit2.3. doi: 10.1002/0471142727.mb0203s27

Robert, L. S., Robson, F., Sharpe, A., Lydiate, D., and Coupland, G. (1998). Conserved structure and function of the Arabidopsis flowering time gene CONSTANS in Brassica napus. Plant Mol. Biol. 37, 763-772. doi: 10.1023/A:1006064514311

Schiessl, S., Iniguez-Luy, F., Qian, W., and Snowdon, R. (2015). Diverse regulatory factors associate with flowering time and yield responses in winter-type Brassica napus. BMC Genomics 16:737. doi: 10.1186/s12864-015-1950-1

Shi, J., Li, R., Qiu, D., Jiang, C., Long, Y., Morgan, C., et al. (2009). Unraveling the complex trait of crop yield with quantitative trait loci mapping in Brassica napus. Genetics 182, 851-861. doi: 10.1534/genetics.109.101642

Shi, J., Zhan, J., Yang, Y., Ye, J., Huang, S., Li, R., et al. (2015). Linkage and regional association analysis reveal two new tightly-linked major-QTLs for pod number and seed number per pod in rapeseed (Brassica napus L.). Sci. Rep. 5, 14481. doi: $10.1038 /$ srep 14481

Tadege, M., Sheldon, C. C., Helliwell, C. A., Stoutjesdijk, P., Dennis, E. S., and Peacock, W. J. (2001). Control of flowering time by FLC orthologues in Brassica napus. Plant J. 28, 545-553. doi: 10.1046/j.1365-313X.2001.01182.x

Uptmoor, R., Li, J., Schrag, T., and Stützel, H. (2012). Prediction of flowering time in Brassica oleracea using a quantitative trait loci-based phenology model. Plant Biol. 14, 179-189. doi: 10.1111/j.1438-8677.2011.00478.x

Van Inghelandt, D., Melchinger, A. E., Martinant, J. P., and Stich, B. (2012). Genome-wide association mapping of flowering time and northern corn leaf blight (Setosphaeria turcica) resistance in a vast commercial maize germplasm set. BMC Plant Biol. 12:56. doi: 10.1186/1471-2229-12-56

Wang, J., Long, Y., Wu, B., Liu, J., Jiang, C., Shi, L., et al. (2009). The evolution of Brassica napus FLOWERING LOCUS T paralogues in the context of inverted chromosomal duplication blocks. BMC Evol. Biol. 9:271. doi: 10.1186/14712148-9-271

Wang, N., Li, F., Chen, B., Xu, K., Yan, G., Qiao, J., et al. (2014). Genome-wide investigation of genetic changes during modern breeding of Brassica napus. Theor. Appl. Genet. 127, 1817-1829. doi: 10.1007/s00122-0142343-6

Wang, N., Qian, W., Suppanz, I., Wei, L., Mao, B., Long, Y., et al. (2011). Flowering time variation in oilseed rape (Brassica napus L.) is associated with allelic variation in the FRIGIDA homologue BnaA.FRI.a. J. Exp. Bot. 62, 5641-5658. doi: $10.1093 /$ jxb/err249

Wang, N., Xiang, Y., Fang, L., Wang, Y., Xin, H., and Li, S. (2013). Patterns of gene duplication and their contribution to expansion of gene families in grapevine. Plant Mol. Biol. Rep. 31, 852-861. doi: 10.1007/s11105-013-0556-5

Wu, J., Wei, K., Cheng, F., Li, S., Wang, Q., Zhao, J., et al. (2012). A naturally occurring InDel variation in BraA.FLC.b (BrFLC2) associated with flowering time variation in Brassica rapa. BMC Plant Biol. 12:151. doi: 10.1186/14712229-12-151

Xu, L., Hu, K., Zhang, Z., Guan, C., Chen, S., Hua, W., et al. (2016). Genomewide association study reveals the genetic architecture of flowering time in rapeseed (Brassica napus L.). DNA Res. 23, 43-52. doi: 10.1093/dnares/ dsv035

Yang, J., Lee, S. H., Goddard, M. E., and Visscher, P. M. (2011). GCTA: a tool for genome-wide complex trait analysis. Am. J. Hum. Genet. 88, 76-82. doi: 10.1016/j.ajhg.2010.11.011

Zou, X., Suppanz, I., Raman, H., Hou, J., Wang, J., Long, Y., et al. (2012). Comparative analysis of FLC homologues in Brassicaceae provides insight into their role in the evolution of oilseed rape. PLOS ONE 7:e45751. doi: 10.1371/journal.pone.0045751

Zuellig, M. P., Kenney, A. M., and Sweigart, A. L. (2014). Evolutionary genetics of plant adaptation: insights from new model systems. Curr. Opin. Plant Biol. 18C, 44-50. doi: 10.1016/j.pbi.2014.01.001

Conflict of Interest Statement: The authors declare that the research was conducted in the absence of any commercial or financial relationships that could be construed as a potential conflict of interest.

The reviewer (NE) and handling Editor declared their shared affiliation, and the handling Editor states that the process nevertheless met the standards of a fair and objective review.

Copyright (c) 2016 Wang, Chen, Xu, Gao, Li, Qiao, Yan, Li, Li and Wu. This is an open-access article distributed under the terms of the Creative Commons Attribution License (CC BY). The use, distribution or reproduction in other forums is permitted, provided the original author(s) or licensor are credited and that the original publication in this journal is cited, in accordance with accepted academic practice. No use, distribution or reproduction is permitted which does not comply with these terms. 